e-J. Surf. Sci. Nanotech. Vol. 3 (2005) 94-96

Conference - Nano-org. \& Func. -

\title{
Scanning Nearfield Optical Spectral Mapping of Cyanine Aggregates on Mica*
}

\author{
Satoshi Nagata, Kazuaki Kaga, and Olaf Karthaus ${ }^{\dagger}$ \\ Chitose Institute of Science and Technology, Bibi 758-65, Chitose 066-8655, Japan \\ (Received 27 December 2004; Accepted 28 February 2005; Published 10 March 2005)
}

\begin{abstract}
Fluorescence microscopy and scanning nearfield optical microscopy and spectroscopy were used to characterize cyanine dyes adsorbed to mica surfaces. The formation of J-aggregates was confirmed, and furthermore it was found that the J-aggregates show a fluorescence-intensity dependent spectral shift. Brighter, and thus larger aggregates show a red-shift in fluorescence spectra of up to $3 \mathrm{~nm}$. [DOI: 10.1380/ejssnt.2005.94]

Keywords: J-aggregate; SNOM; size-effect
\end{abstract}

\section{INTRODUCTION}

Thin films of organic materials are often required for many applications in photonic science. Dye aggregates or dye crystals often show different characteristics (absorption and fluorescence spectra, excited state lifetime, excited state relaxation, etc.) than the molecularly dispersed or amorphous state. Thus it is of utmost importance to control the aggregation state and to measure the photonic response.

It is known since the 1930ies that cyanine dyes can be used as photosensitizers in photographic films [1]. Cyanine dyes form so-called J-aggregates in solution and in spin-coated polymer films. J-aggregates show a characteristic red-shift of absorption and fluorescence spectra. Furthermore, the spectral width is only $10 \sim 20 \mathrm{~nm}$, which is much narrower than the molecular dispersed state.

The spectral shape and the absorption and fluorescence
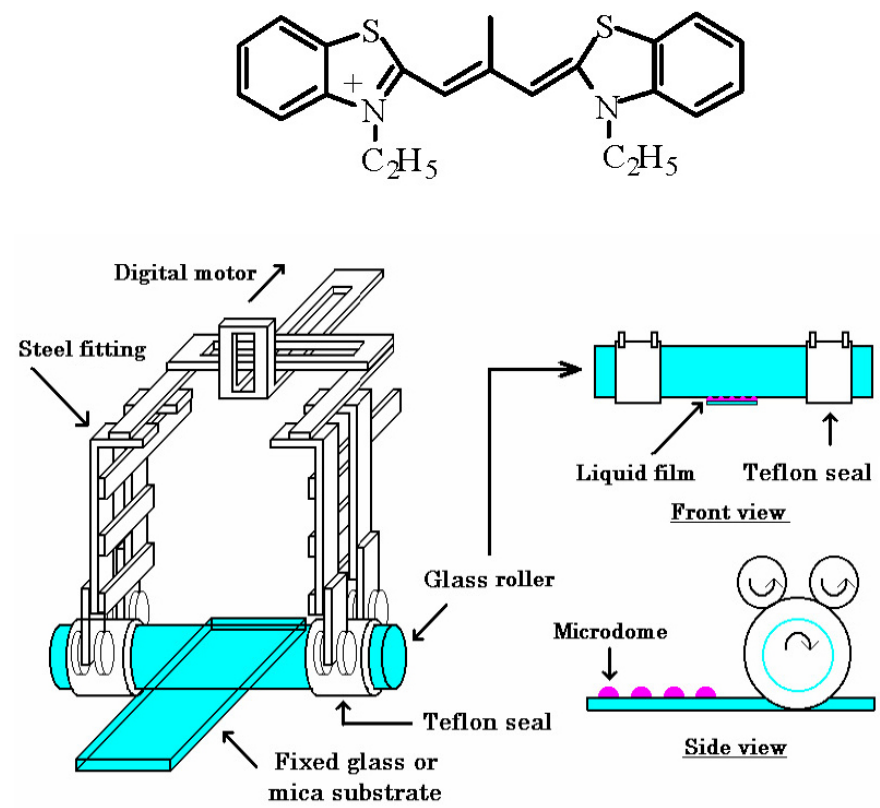

FIG. 1: Schematic drawing of the roller apparatus and chemical formula of the cyanine dye.

\footnotetext{
* This paper was presented at International Symposium on Nanoorganization and Function, Tokyo, Japan, 11-12 November, 2004. †Corresponding author: karthaus@photon.chitose.ac.jp
}

maxima can be controlled by confining J-aggregates into micronsized polymer droplets, so-called 'microdomes' [2, $3]$.

Here we report on the adsorption of cyanine dyes onto mica surfaces and in polyer microdomes and the spectral shift of the resulting J-aggregates depending on the aggregate fluorescence intensity. Scanning nearfield optical microscopy (SNOM) was used, since it allows for a submicrometer resolution. Previous experiments [2, 3] used far field microscopy with a lateral resolution of $2 \mu \mathrm{m}$. Since the polymer domes have a spacing larger than that, individual microdomes can be investigated. In the case of adsorption of the dye onto mica, the aggregate density is higher and thus a better lateral resolution is necessary. Furthermore, the topography of the sample can be recorded simultaneously, and not in to separate measurements, as in the case of far field microspectrometry.

\section{EXPERIMENTAL}

The cyanine dye (N, N'-diethyl-9methylthiacarbocyanine iodide, NK77, see Fig. 1) (Aldrich) was dissolved in chloroform (p.a. Merck, Germany) and approx. $100 \mu \mathrm{l}$ of a $0.2 \mathrm{mM}$ solution was placed close to the edge of a glass slide or a freshly cleaved mica substrate of $2 \mathrm{~cm} \times 5 \mathrm{~cm}$. A glass roller was used to cast the solution uniformly over the whole

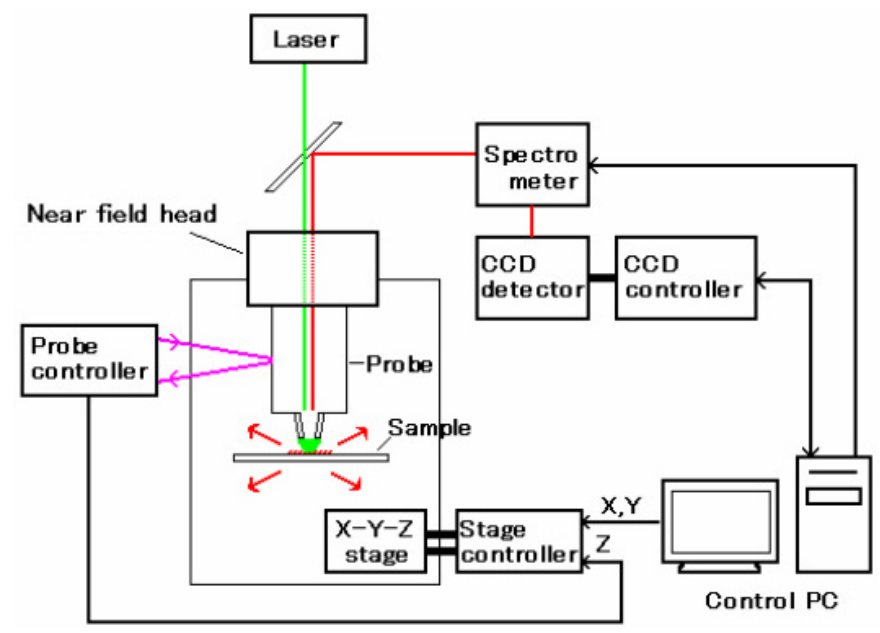

FIG. 2: Schematic of the scanning nearfield optical microscope setup. 


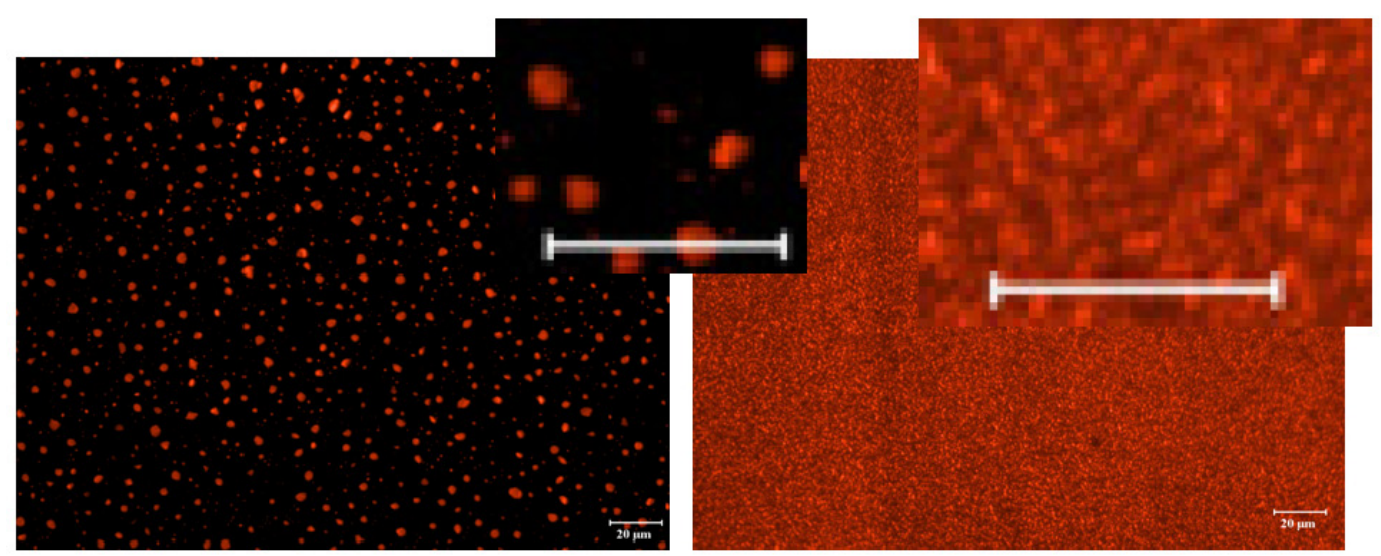

FIG. 3: Fluorescence micrograph (green excitation) of NK77 (see Figure 1) thin film on glass (left) and on muscovite mica (right). The insets are magnified areas of the samples. The scale bars are $20 \mu \mathrm{m}$ in all cases.
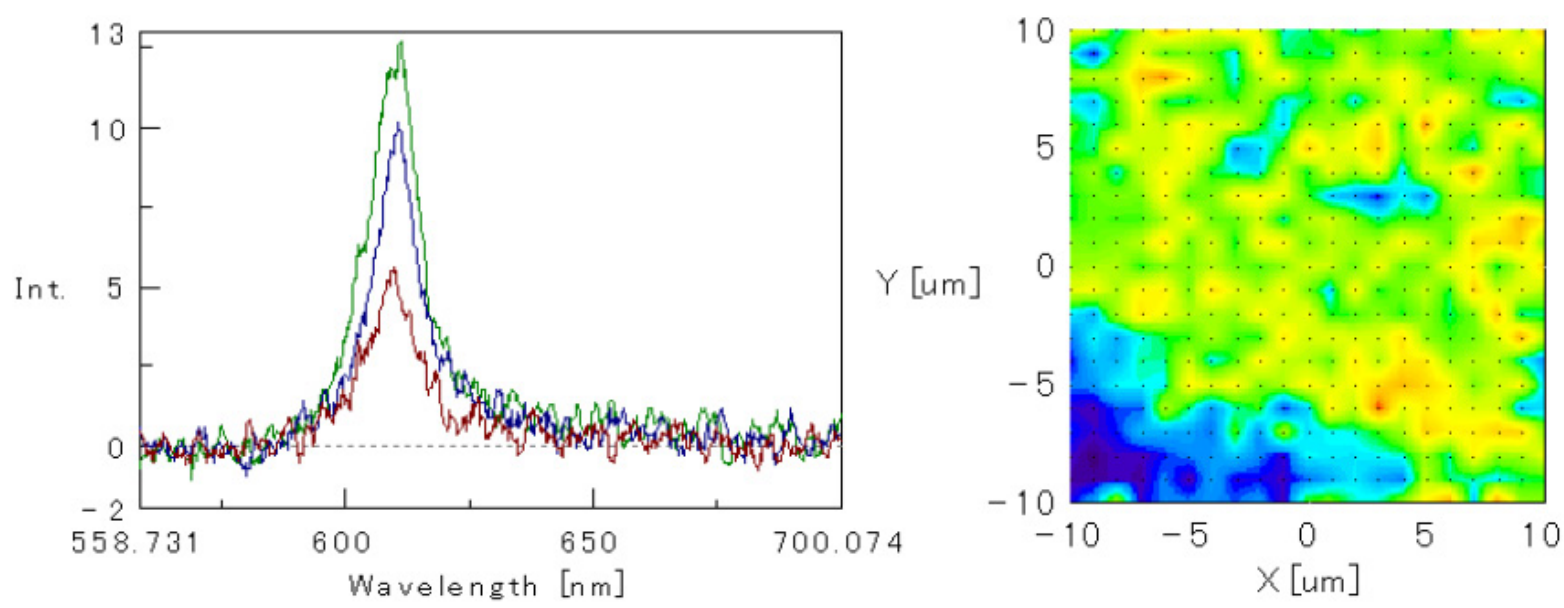

FIG. 4: Fluorescence spectra of cyanine J-aggregates on mica substrate taken at different places within a $20 \mu \mathrm{m} \times 20 \mu \mathrm{m}$ area (left). Map of $21 \times 21$ fluorescence spectra taken of a $20 \mu \mathrm{m} \times 20 \mu \mathrm{m}$ area (right). The color scale encodes the relative intensity of the spectra (blue is low, red is high intensity).

substrate. The roller speed was $5 \mathrm{~mm} / \mathrm{min}$ and 10 $\mathrm{mm} / \mathrm{min}$, respectively. Fluorescence microscopic images were obtained with an Olympus BX-51 microscope equipped with a color CCD camera (SPOT-RT, Diagnostics Inc.). Scanning nearfield optical micrographs were taken under illumination/collection mode (NFS-230K, JASCO, Japan, see Fig. 2) in which the sample was excited through a fiber (aperture $410 \mathrm{~nm}$ ) with laser light of $532 \mathrm{~nm}$. The fluorescence was collected by the same fiber and the fluorescence spectrum was recorded for each spot of a $21 \times 21$ point matrix, covering $20 \mu \mathrm{m} \times 20 \mu \mathrm{m}$ of the sample. The spectral resolution was $<0.19 \mathrm{~nm}$.

\section{RESULTS}

Figure 3 shows the fluorescence micrographs of NK77 adsorbed onto glass and freshly cleaved muscovite mica. The excitation wavelength was $440 \sim 480 \mathrm{~nm}$ and the fluorescence emission $>520 \mathrm{~nm}$ was detected, by using a far field fluorescence microscope. The glass sample shows a non-uniform coverage of the surface with large aggregates having a large variation in brightness. In contrast, the mica surface is homogeneously covered with aggregates with diameters of $1 \sim 3 \mu \mathrm{m}$. It is known that cationic cyanine can adsorb onto the negatively charged mica surface to form J-aggregates [4]. Direct investigation of the dye aggregates by atomic force microscopy showed epitaxial growth of the aggregates with an aggregate height of a few nanometers [5].

First evidence for the effect of the J-aggregate size on the photophysical properties have been obtained from conventional far-field microspectrosopy of microscopic mica flakes, on which J-aggregates were adsorbed [6]. It was found that larger flakes of a diameter of around 10 $\mu \mathrm{m}$ give rise to a red-shifted fluorescence, as compared to flakes with a diameter of $1 \sim 3 \mu \mathrm{m}$. Furthermore, the Jaggregates on larger mica flakes were more stable against photobleaching. Both findings led to the conclusion that larger flakes produce larger J-aggregates, and that the larger J-aggregates are more stable and show red-shifted fluorescence [6].

Figure 4 shows the mapping of a $20 \mu \mathrm{m} \times 20 \mu \mathrm{m}$ area of a large mica substrate, which is covered with J-aggregates 
TABLE I: Spectral characteristics of the three spectra in Fig. 4. Position refers to the position of the spectrum in Fig. 4.

\begin{tabular}{cccc}
\hline \hline Position $(x, y)$ & peak $(\mathrm{nm})$ & peak intensity (arb. unit.) & half-width (nm) \\
\hline$(3,-6)$ & & & 11.3 \\
$(3,6)$ & 611.1 & 13 & 10.0 \\
$(-7,3)$ & 610.7 & 10 & 12.1 \\
\hline \hline
\end{tabular}

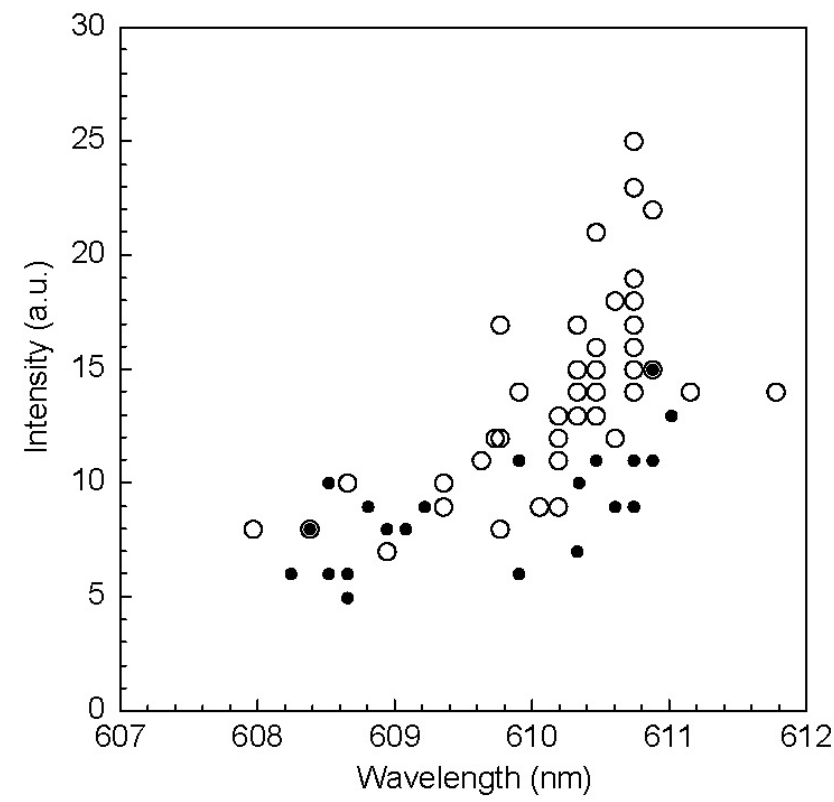

FIG. 5: Plot of the fluorescence intensity against the peak wavelength of two samples: roller speed $5 \mathrm{~mm} / \mathrm{min}$ (solid dots) and $10 \mathrm{~mm} / \mathrm{min}$ (open dots).

(as seen in Fig. 2). The image was obtained by measuring 441 fluorescence spectra in a square $21 \times 21$ point matrix. The image was reconstructed by plotting the maximum fluorescence intensity of each spectrum at its corresponding position. Fluorescence peaks were found between 608 and $612 \mathrm{~nm}$. Fluorescence intensities varied between zero and thirteen counts per second. The micron-size inhomogeneities correspond very well with the fluorescence micrograph of Fig. 1. The spectra show the typical redshifted fluorescence and a narrow shape with a full width at half maximum of about $10 \mathrm{~nm}$, which is characteristic for J-aggregates. Three of the 441 spectra are also shown in Fig. 4.
The characteristics (maximum fluorescence wavelength, peak intensity and spectral width) of the three spectra are summarized in Table 1. There is a correlation between fluorescence intensity (inferred from the peak height) and the maximum fluorescence wavelength. Albeit small, there is a distinct shift of the fluorescence to longer wavelengths for brighter, and thus larger aggregates. The half width of the spectra seems not to be correlated with the peak intensity.

Figure 5 shows the correlation between peak intensity and peak wavelength for several spectra for two samples: $5 \mathrm{~mm} / \mathrm{min}$ roller speed and $10 \mathrm{~mm} / \mathrm{min}$.

It is evident that the spectra shift to longer wavelength for both samples, even though the effect is more pronounced for the sample prepared at higher roller speed. It has already been reported that the fluorescence spectra of cyanine dye J-aggregates embedded in polymer 'microdomes' show a similar effect. Larger domes lead to red-shifted aggregates. This can be explained by the formation of larger J-aggregates in larger domes, since there are more molecules in the larger domes that can diffuse and aggregate. Thus it was possible to tailor the emission wavelength of a fluorescent material by changing the size of the physical confinement into micrometer-sized domes. In the present results, the size of the aggregates cannot be exactly determined yet. Still, the size was inferred from the fluorescence intensity and a similar effect of the dependence of fluorescence wavelength and emission maximum was found.

This new finding confirms that the fluorescence shift with aggregate size is not a measurement artefact due to re-absorption of fluorescence light by the J-aggregates in the three dimensional polymer microdomes. The Jaggregates on mica are known to have a thickness of just a few molecules, which rules out re-absorption in this quasi two-dimensional geometry. Furthermore, the illumination/collection mode of the SNOM also ensures no re-absorption of the fluorescence light.
[1] G. Scheibe, Angew. Chem. 49, 563 (1936).

[2] K. Kaga, K. Okamoto, T. Echizen, O. Karthaus, K. Nakajima, Kobunshi Ronbunshu 60, 752 (2003).

[3] O. Karthaus, K. Kaga, J. Sato, S. Kurimura, K. Okamoto, T. Imai, in Nonlinear Dynamics in Polymeric Systems, ACS Symposium Series No. 869, J. A. Pojman and Q. TranCong-Miyata, Eds. (American Chemical Society, Washing- ton, DC), pp 199-211 (2004).

[4] G. Scheibe, Angew. Chem. 52, 631 (1939).

[5] S. Sugiyama, H. Yao, O. Matsuoka, R. Kawabata, N. Kitamura, S. Yamamoto, Chem. Lett. 1999, 37 (1999).

[6] O. Karthaus, Y. Kawatani, Jpn. J. Appl. Phys. 42, 127 (2003). 